\title{
ATLAS Silicon Microstrip Tracker Operation and Performance
}

\author{
Peter Vankov \\ On behalf of the ATLAS collaboration
}

\begin{abstract}
In December 2009 the ATLAS experiment at the CERN Large Hadron Collider (LHC) recorded the first protonproton collisions at a center-of-mass energy of $900 \mathrm{GeV}$ and this was followed by the unprecedented energy of $7 \mathrm{TeV}$ in March 2010. The SemiConductor Tracker (SCT) is the key precision tracking device in ATLAS, made up from silicon microstrip detectors processed in the planar p-in-n technology. The completed SCT has been installed inside the ATLAS experimental hall. After the commissioning phase it arrived to the first LHC pp collision runs in very good shape: $\mathbf{9 9 . 3 \%}$ of the SCT modules are operational, noise occupancy and hit efficiency exceed the design specifications, the alignment is already close enough to the ideal one to allow on-line track reconstruction and invariant mass determination.

This overview presents the current status of the SCT, including results from the latest data-taking periods in 2009 and 2010, and from the detector alignment. We report on the operation of the detector and observed problems. The main emphasis is given to the performance of the SCT with the LHC in collision mode in a comparison with the expected parameters and with the MonteCarlo simulations.
\end{abstract}

\section{INTRODUCTION}

The "A Toroidal LHC ApparatuS" (ATLAS) [1] is a general-purpose high-energy physics experiment designed to explore the proton-proton collisions at the Large Hadron Collider (LHC) [2]. The LHC at the European Laboratory for Particle Physics, CERN, in Geneva, Switzerland is currently the world's most powerful particle accelerator. Placed $100 \mathrm{~m}$ underground in a $27 \mathrm{~km}$ tunnel, LHC is designed to collide protons with a center-of-mass energy of $\sqrt{s}=14 \mathrm{TeV}$ at a maximum luminosity of the order of $10^{34} \mathrm{~cm}^{-2} \mathrm{~s}^{-1}$. The unprecedentedly high collision energy along with the high luminosity at the LHC will eventually allow discovery of possible new physics effects at the $\mathrm{TeV}$ scale. ATLAS is constructed to exploit the full physics potential of LHC but will mainly focus on the discovery of the still unobserved Higgs particle, and on searches of supersymetry and extradimensions. The expected ATLAS physics discovery performance is presented in detail in Ref. [3].

A cut-away view of the ATLAS detector is shown in Fig. 1. With overall dimensions of $44 \mathrm{~m}$ in length and $25 \mathrm{~m}$ in diameter, ATLAS is the largest ever built high-energy physics experiment. It comprises three basic subsystems: the Inner Detector, housed in a solenoid creating magnetic field of $2 \mathrm{~T}$, the Calorimetry system (hadronic and electromagnetic) and

P. Vankov is with the Deutsches Elektronen Synchrotron, DESY at Hamburg, Germany.

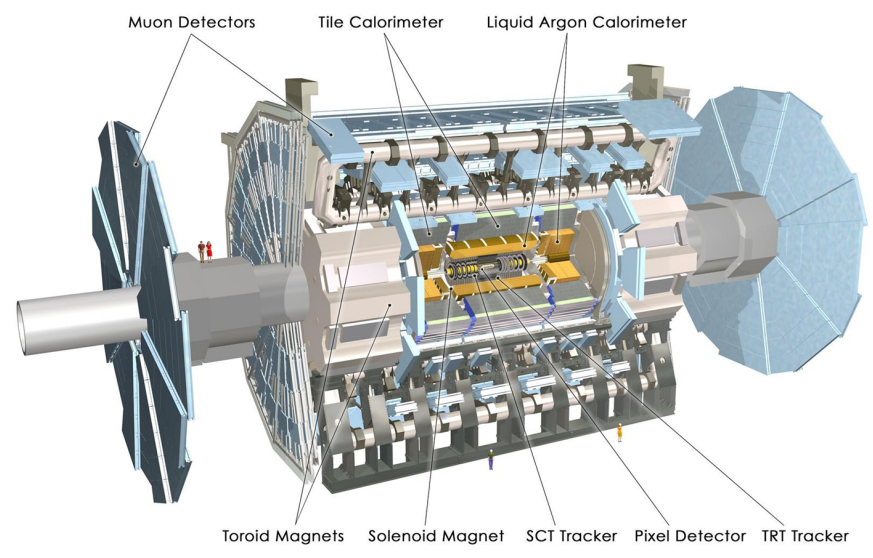

Fig. 1. The ATLAS experiment.

the Muon Spectrometer with its associated superconducting toroidal magnets applying magnetic field of $0.5 \mathrm{~T}$.

The Inner Detector (ID) [4] of ATLAS is optimized to provide excellent momentum $\left(\sigma_{p_{T}} / p_{T} \sim 0.05 \% p_{T}(\mathrm{GeV}) \oplus 1 \%\right)$ and vertex $\left(\sigma_{d_{0}} \sim 10 \mu \mathrm{m}\right)$ resolutions for particles with pseudo-rapidity $|\eta| \leq 2.5$. In addition, to cope with the high interaction rates and particle fluxes at the interaction region the ID is devised to incorporate high granularity, radiation hardness and fast responsiveness. As presented in Fig. 2, the ID is composed of three complementary subsystems situated inside the $2 \mathrm{~T}$ solenoid: the Pixel detector, forming the inmost, closest to the interaction point layer of the ID, the SemiConductor Tracker (silicon microstrips) occupying the central ID region, and the Transition Radiation Tracker (TRT) (arrays of gaseous straw drift-tubes), at the external ID side. Each of the three ID components is built of a central barrel section and two end-caps in the forward regions.

\section{The SemiConductor Tracker}

The SemiConductor Tracker of the ATLAS experiment has a modular design. It is built of 4088 silicon modules arranged in 4 concentric, barrel layers, and 18 end-cap discs. In total, SCT integrates area of $61 \mathrm{~m}^{2}$ of silicon micro-strip sensors with 6.3 million readout channels. The SCT barrel part, consisting of 2112 modules, covers pseudo-rapidity region of $|\eta|<1.1$ to 1.4, depending on the layer, whereas the end-caps, with 1976 modules in total, extends this region up to $|\eta|<2.5$. The radius of the innermost barrel is $30 \mathrm{~cm}$ and the outermost barrel layer as well as the end-caps have radius of $56 \mathrm{~cm}$. Along the beam direction (the $z$-axis), the length of the SCT 


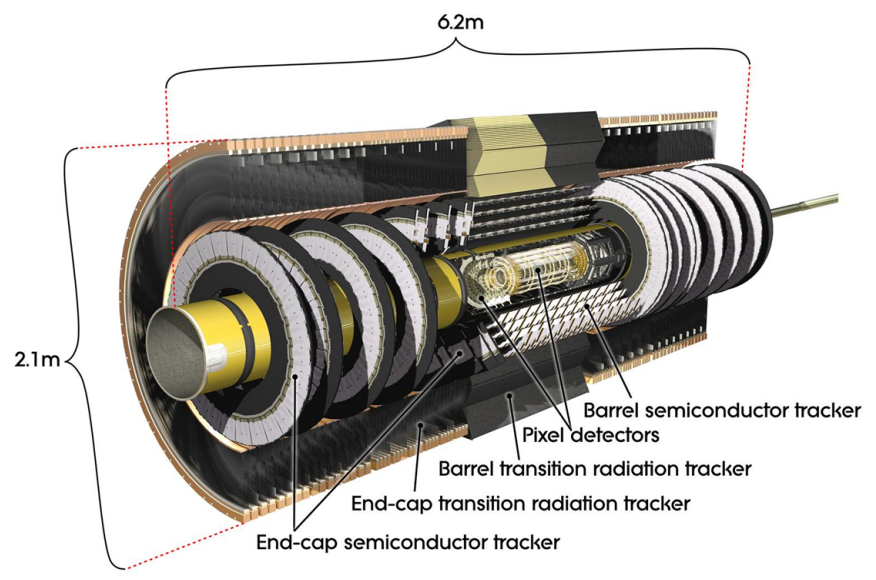

Fig. 2. The Inner Detector of ATLAS.

barrel, centered at the interaction point (IP), is $160 \mathrm{~cm}$. The two groups of 9 end-cap discs, identified as end-cap $A$ and end-cap $C$ are symmetrical relative to the IP. Along $z$, they are positioned on a distance of $85 \mathrm{~cm}$ to $272 \mathrm{~cm}$ away from the IP.

The general layout of the barrel and end-cap SCT modules is similar; the difference is primarily in the module's shape. The barrel modules are identical [5], whereas the end-cap ones come in 4 different types [6]. All SCT modules are built of 2 pairs of silicon (p-in-n) microstrip sensors, glued back-toback at an angle of $40 \mathrm{mrad}$ to provide $2 \mathrm{D}$ space-points, see Fig. 3. There are 768 silicon strips per module side (1536 per module) at a pitch of $80 \mu \mathrm{m}$ for the barrel and from $57 \mu \mathrm{m}$ to $94 \mu \mathrm{m}$ for the end-cap modules. A nominal bias voltage of $150 \mathrm{~V}$ is applied to the silicon strips. Full depletion is achieved at $65 \mathrm{~V}$. The module power consumption is $5.6 \mathrm{~W}$ (without irradiation). This module architecture allows achievement of space-point resolution of $17 \mu \mathrm{m}$ in the $R \phi$ and $580 \mu \mathrm{m}$ in $z$ directions.

The readout of the SCT modules is performed by six 128channel ADCD3TA chips [7] on each side of the module, fabricated in the radiation hard technology DMILL. The data signals, processed by the chips are pre-amplified, shaped,

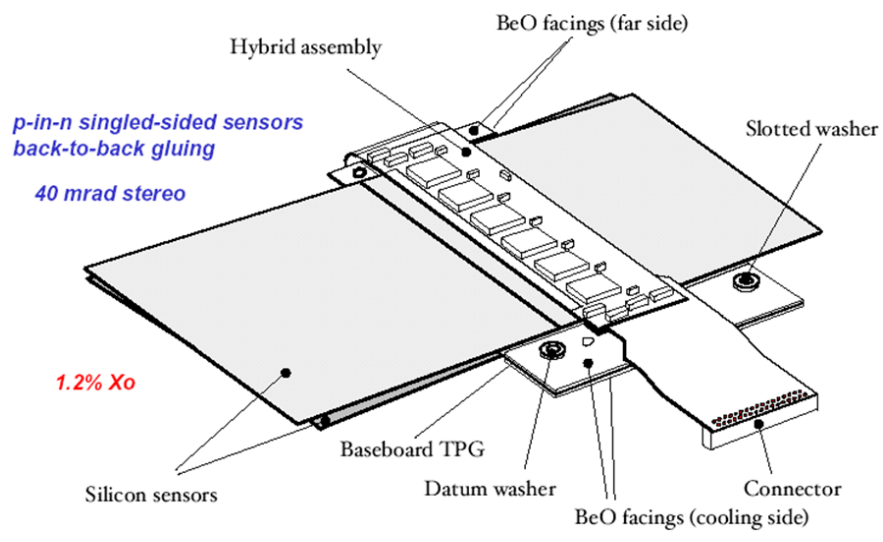

Fig. 3. A drawing of the SCT barrel module. discriminated (compared to a nominal threshold of $1 \mathrm{fC}$ ) and finally digitized; binary output is produced. The communication of the module with the off-detector electronics is realized through optical links, VCSELs, operating at $40 \mathrm{Mbits} / \mathrm{s}$ for the data readout. The opto-electronics used for this includes VDC chip [8] (drives the laser diodes) and DORIC4A chip [8] (receives the clock and command data from the light-sensitive diode).

For a successful 10-years operation at the harsh radiation environment at LHC, the SCT modules must withstand a $1 \mathrm{MeV}$ neutron equivalent fluence of $2 \times 10^{14} \mathrm{~cm}^{2}$. To limit the radiation damage effects, such as reverse annealing and leakage current, and to decrease the noise levels, the SCT detector is cooled down to $-7^{\circ} \mathrm{C}$ (the end-caps), $-2^{\circ} \mathrm{C}$ to $-1.5^{\circ} \mathrm{C}$ (the 3 inner barrel layers) and $4.5^{\circ} \mathrm{C}$ (the 4 th outer barrel). The cooling is performed by evaporative $\mathrm{C}_{3} \mathrm{~F}_{8}$-based system operating nominally at $-25^{\circ} \mathrm{C}$.

\section{Comissioning, Calibration, Cosmics And COLLISIONS DATA}

The installation and comissioning of the SCT detector took part in the period August 2006 - February 2008. First, the SCT barrel was inserted in the ATLAS cavern in August 2006, then in April 2007 the end-caps were added. Post-installation and commissioning tests took place after the positioning of the SCT. In March 2008 the SCT joined the ATLAS combined M6 Milestone run with most other sub-detector systems and with all trigger levels. After successful integration with the central DAQ, SCT started taking cosmic data.

For the launch of the LHC on $10^{\text {th }}$ of September 2008 with circulating proton beams in both directions at an injection energy of $450 \mathrm{GeV}$, SCT was calibrated and ready for operation. The first detected beam events were caused by splashes of the protons at a collimator close to ATLAS. For safety reasons the SCT barrel was turned off and only the end-caps were left to function at a decreased voltage $(20 \mathrm{~V})$ and a raised threshold (1.2 fC).

After the LHC magnet-failure accident of $19^{\text {th }}$ of September 2008, SCT took part in an extensive ATLAS-global cosmicruns programm, which continued until the re-initiation of the LHC on $20^{\text {th }}$ of November 2009 with single beam splashes. This was followed by the first LHC proton-proton collisions at $\sqrt{s}=900 \mathrm{GeV}$ and later on at $\sqrt{s}=2.36 \mathrm{TeV}$, successfully detected by SCT and ATLAS.

Since $30^{\text {th }}$ of March 2010, LHC is in run phase 1, colliding protons at $\sqrt{s}=7 \mathrm{TeV}$ (3.5 TeV per beam). Large amount of $7 \mathrm{TeV}$ data was collected with SCT on. The total integrated luminosity recorded by ATLAS until 30.10.2010 equals to $45 \mathrm{pb}^{-1}$.

\section{SCT OPERATION}

\section{A. SCT operational status}

Figure 4 shows a summary of the disabled SCT modules, chips and masked strips during the collision runs in 2010 . In total, 30 modules (out of 4088) are excluded of the configuration of the SCT in the 2010 physics data-taking. 


\begin{tabular}{l|ccccc}
\hline $\begin{array}{l}\text { Disabled Readout } \\
\text { Components }\end{array}$ & Endcap A & Barrel & Endcap C & SCT & $\begin{array}{c}\text { Fraction } \\
(\%)\end{array}$ \\
\hline Disabled Modules & 5 & 10 & 15 & 30 & 0.73 \\
Disabled Chips & 5 & 24 & 4 & 33 & 0.07 \\
Masked Strips & 3,364 & 3,681 & 3,628 & 10,673 & 0.17 \\
\hline $\begin{array}{l}\text { Total Disabled } \\
\text { Detector Region }\end{array}$ & & & & & 0.97 \\
\hline
\end{tabular}

Fig. 4. Summary of the SCT disabled modules in 2010.

The reason is mostly in a leaking cooling loop in the endcap C, preventing 13 modules to be turned on. The rest of the modules are not operational due to connection problems with the low and high-voltage power supplies. The 33 disabled chips comprise 31 chips which are bypassed and 2 chips for which all strips are masked. The number of masked strips include strips that are defective or have high noise occupancy. Currently, $99.3 \%$ of the SCT detector is fully functional.

\section{B. Operational issues}

In May 2008 a cooling plant failure occurred, which put the SCT out of operation. The incident affected three out of the six compressors of the ID cooling-plant, which is common for the Pixels and SCT. Three months later, at the end of August 2008 , the damaged compressors were replaced, and since then the cooling is functional.

During operation, sudden failures of the off-detector transmitter boards (Tx-plugins) started to happen. It was believed that this problem is caused by electro-static discharges at the VCSEL boards (used to transmit clock and commands to the modules). All broken VCSELs were replaced before the start of the $7-\mathrm{TeV}$ collisions in March 2010. However, the issue reappeared a few months later. It is under deeper investigation now. For the time being a redundancy schema is used.

Another issue faced was the quality of thermal interface between the SCT barrel and the TRT causing SCT barrel to run at higher temperature.

\section{SCT PERFORMANCE}

\section{A. Timing}

It is important to be sure that the SCT detector is well "timed in" with the trigger signal. Presently, SCT runs in anyhit-mode, i.e, it reads out three $25 \mathrm{~ns}$ time-bins (or 3 LHC bunch-crossings (BX)) around the ATLAS Level-1 trigger. A hit is recorded if the charge deposited on a strip is greater than $1 \mathrm{fC}$ in any of these time-bins. Properly synchronized, the SCT must receive an accept trigger signal in the same $\mathrm{BX}$ as the collision products pass through it. This is achieved by applying delays to the trigger signal in order to account for the time-of-flight of particles and for signal propagation times. If SCT is correctly timed in then the hit-pattern should be: nothing in the first time-bin, over threshold in the second time-bin and no requirement for the third time-bin, which also suggests that the average time-bin should be a value between 1 and 2. In Fig. 5 the average time-bin for all SCT regions is plotted. All values are in the range $[1,2]$, which demonstrates that SCT is accurately and uniformly timed in.

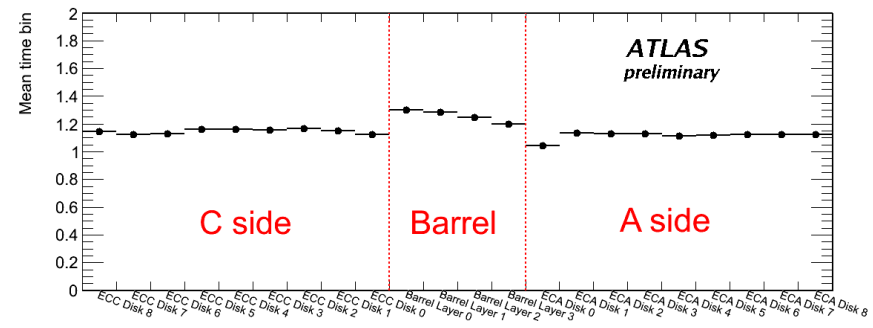

Fig. 5. The average time-bin for the different SCT regions.

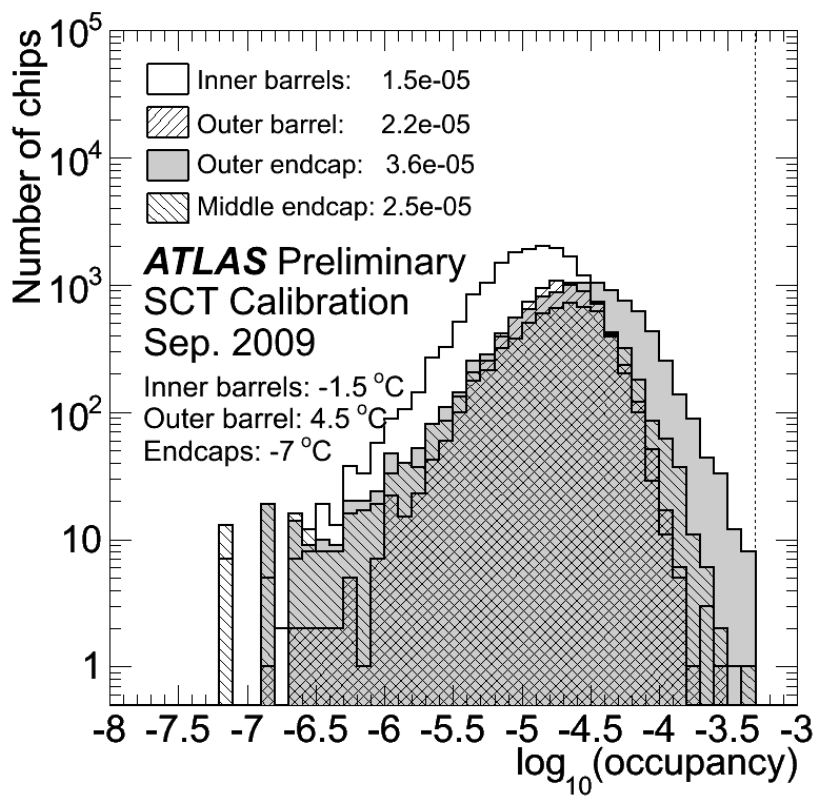

Fig. 6. Noise occupancy averaged over chips in the SCT barrel and endcaps. The specification limit of $5 \times 10^{-4}$ is indicated with dashed line at the right-hand side of the plot.

\section{B. Noise}

The noise performance of the SCT is illustrated in Fig. 6 where the distribution of the $\left(\log _{10}\right.$ of) average noise occupancy per chip obtained with random triggers is presented with nominal values of the threshold $(1 \mathrm{fC})$ and bias voltage $(150 \mathrm{~V})$. The dashed line indicates the TDR [4] noiseoccupancy requirement limit of $5 \times 10^{-4}$. It can be seen that all the measured values are well below this limit. The inner and middle short end-cap modules are not shown since for them the average noise occupancy was below the sensitivity of the performed tests.

\section{Data and Simulation}

The number of fired strips per module side in a $\sqrt{s}=$ $900 \mathrm{GeV}$ pp-collision run for all (barrel + end-caps) SCT modules is presented in Fig. 7. The data is compared to a $900 \mathrm{GeV}$ minimum-bias Monte-Carlo (MC)-simulated sample.

Both distributions are normalized to the number of events. Good agreement between data and MC is found for a wide range of number of strips. The discrepancy observed in the first bin is attributed to the lower levels of noise implemented 


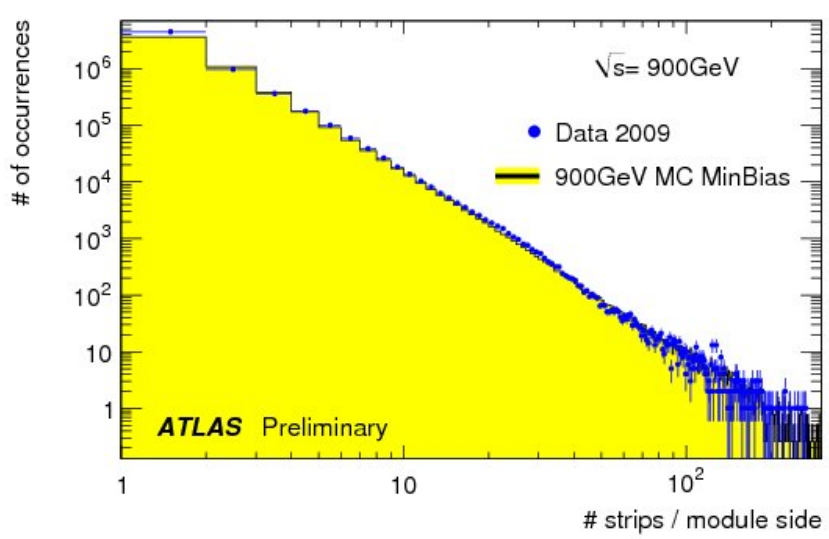

Fig. 7. Number of SCT hit-strips in a $\sqrt{s}=900 \mathrm{GeV}$ pp run compared to a $900 \mathrm{GeV}$ minimum-bias Monte-Carlo simulated sample.

in the MC (the MC is simulated with only one BX, while the data is with three BX's).

\section{Efficiency}

Figure 8 shows the intrinsic hit-efficiency of the SCT barrel. The efficiency is extracted using reconstructed tracks. The tracks used, were required to have at least 6 SCT hits and at least 20 TRT hits. The efficiency is then derived as the ratio of the number of hits on a track and the number of expected hits. Disabled modules and chips are not counted and are excluded from the measurement. On average the barrel hit-efficiency is found to be $99.8 \% \pm 0.1 \%$. The end-caps showed similar hit-efficiency values.

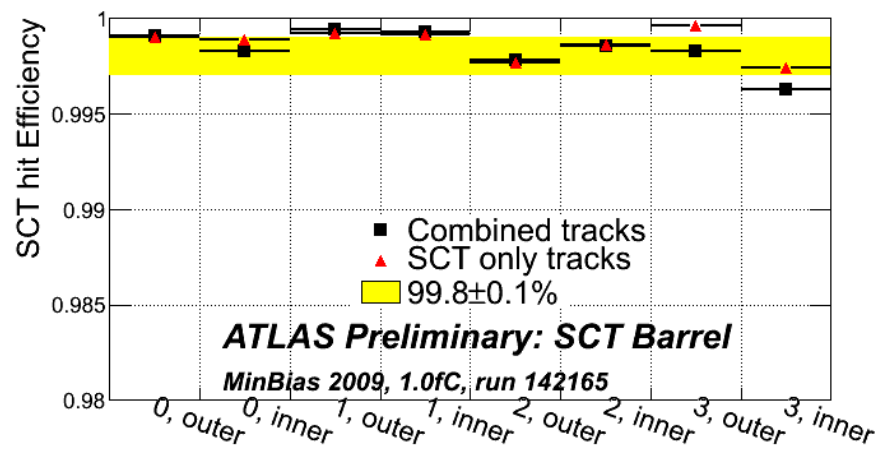

Fig. 8. SCT barrel hit-efficiency (4 layers, 2 sides - inner and outer).

\section{E. Lorentz angle}

Another important quantity measured was the Lorentz angle, $\theta_{L}$. This is the angle by which charge carriers in the Si-sensor drift in presence of magnetic field. The Lorentz angle can be defined by measuring the track incidence angle leading to a minimum cluster-size (number of strips in a cluster). The measured mean cluster-size as a function of the incidence angle, in degrees, is plotted in Fig. 9 for the four layers of the SCT barrel. The Lorentz angle is then obtained by the

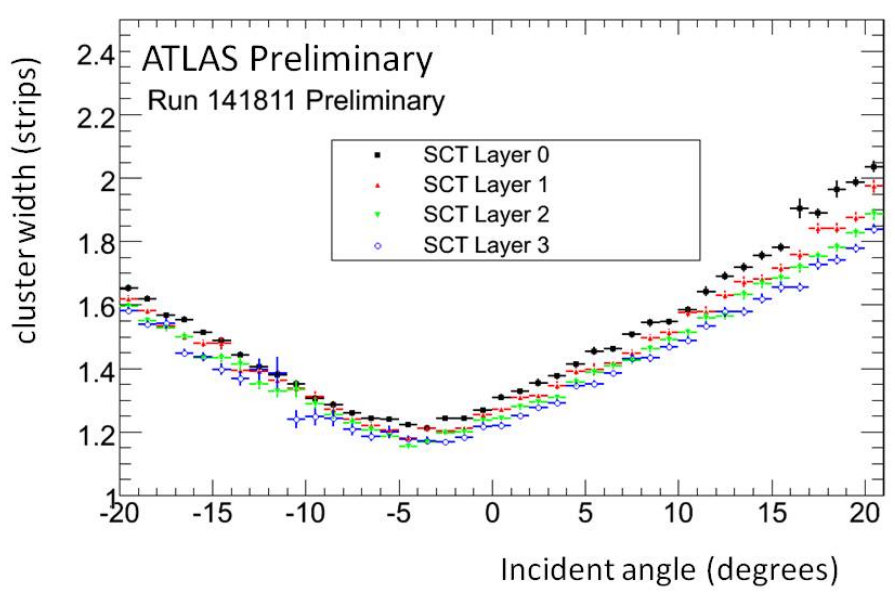

Fig. 9. Mean cluster size as a function of the track incidence angle for the SCT barrel layers.

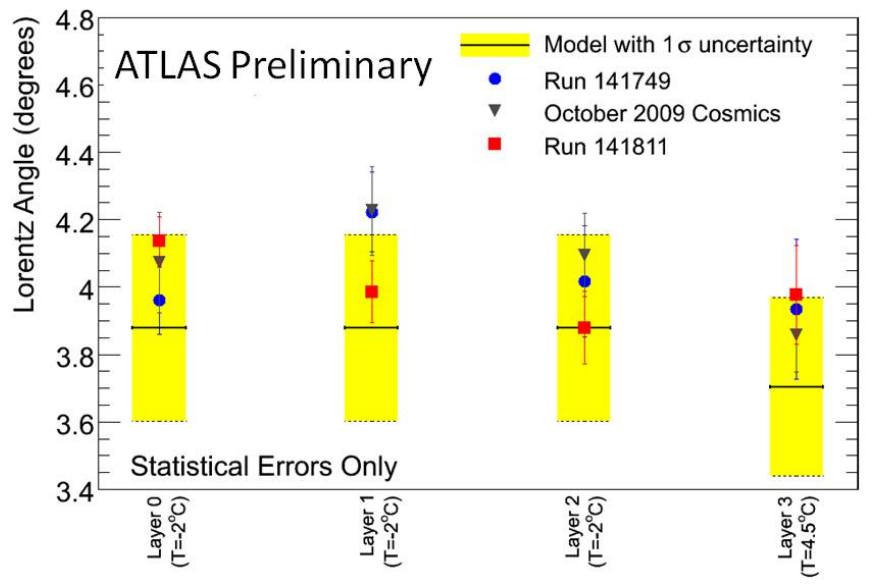

Fig. 10. The Lorentz angle defined from one cosmic and two $900 \mathrm{GeV}$ runs, compared to the model prediction. SCT barrel.

position of the minimum of the distribution. Figure 10 shows the so obtained $\theta_{L}$ for two $900-\mathrm{GeV}$ collision-runs and one 2009 cosmic-run compared to the model-prediction value. As can be seen, the results for $\theta_{L}$ are in good agreement (within $1 \sigma$ ) with the model prediction. When there is no magnetic field applied, the Lorentz angle is found to be in the vicinity of 0 degrees, as expected.

\section{Alignment}

The SCT alignment for 2010 was performed using the collected in 2009 collision data-sets at $\sqrt{s}=900 \mathrm{GeV}$. The alignment is already very close to the ideal one as demonstrated in Fig. 11 (barrel) and Fig. 12 (end-caps), where the $x$ track-residual distributions are shown for $7-\mathrm{TeV}$ collision data compared to Monte-Carlo simulation with nominal alignment. The residuals are constructed as the difference between the measured $x$ hit-position and the expected $x$ position, as the latter is obtained via track extrapolation. The used tracks are reconstructed with the requirement of having at least $6 \mathrm{SCT}$ hits and $p_{T}>2 \mathrm{GeV}$. From the plots it is evident that the SCT is well aligned and its geometry approximates closely 


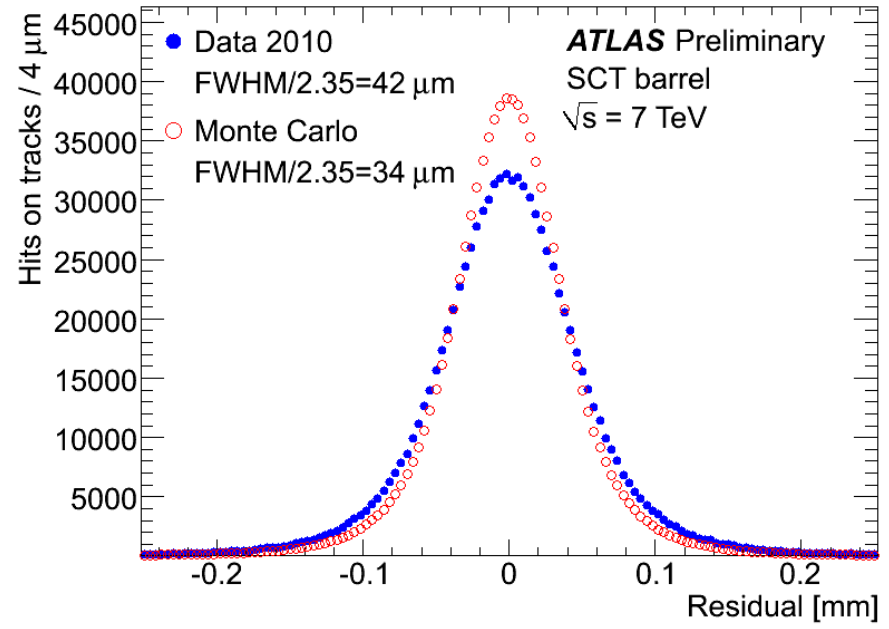

Fig. 11. Track $x$-residual distribution for SCT barrel with perfect (MC) geometry (in red) and for the current alignment with $7-\mathrm{TeV}$ collision data (in blue).

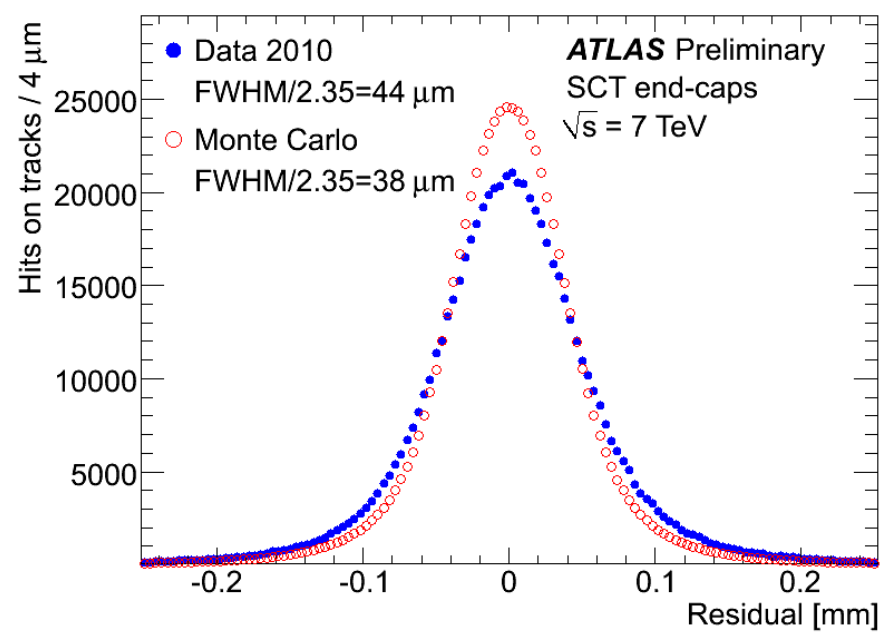

Fig. 12. Track $x$-residual distribution for SCT end-caps with perfect (MC) geometry (in red) and for the current alignment with $7-\mathrm{TeV}$ collision data (in blue).

the perfect, Monte-Carlo-simulated geometry. This is of great importance for performing accurate tracking and invariant mass reconstructions. The reconstructed invariant mass of the $K_{s}^{0}$ meson is shown in Fig. 13 as an example.

\section{CONCLUSION}

The ATLAS SemiConductor Tracker is fully functional, with more than $99 \%$ of operational modules. Its performance is more than satisfactory, showing moderate noise-levels, high intrinsic hit-efficiency and good agreement with the MCsimulation description. Well aligned, the detector is in excellent condition and meets the design specifications. It has been successfully used in the 2009 and 2010 collision data-taking.

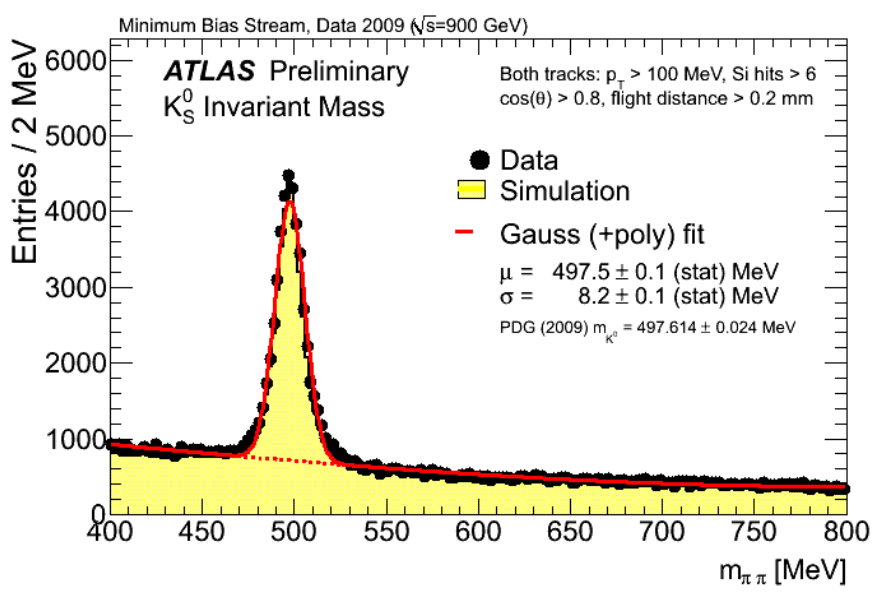

Fig. 13. $K_{s}^{0}$ invariant mass.

\section{REFERENCES}

[1] G. Aad, et al., The ATLAS Experiment at the CERN Large Hadron Collider, J. Instr. 3 (2008) S08003.

[2] L. Evans and P. Bryant, LHC Machine, J. Instr. 3 (2008) S08001.

[3] The ATLAS Collaboration, Expected Performance of the ATLAS Experiment - Detector, Trigger and Physics, arXiv:0901.0512v4 (2009).

[4] ATLAS Collaboration, ATLAS Inner Detector Technical Design Report, Volume 2, ATLAS TDR 5, CERN/LHCC/97-17, ISBN 92-9083-103-0.

[5] A. Abdesselam, et al., The Barrel Modules of the ATLAS Semiconductor Tracker, Nucl. Instrum. Meth. A568 (2006), 642.

[6] A. Abdesselam, et al., The ATLAS semiconductor tracker end-cap module, Nucl. Instrum. Meth. A575 (2007), 353.

[7] F. Campabadal, et al., The Design and performance of ABCD3TA ASIC for readout of silicon strip detectors in the ATLAS semiconductor tracker, Nucl. Instrum. Meth. A552 (2005), 292.

[8] D.J. White, et al., Radiation hardness studies of the front end ASICs for the optical links of the ATLAS SemiConductor Tracker, Nucl. Instrum. Meth. A457 (2001), 369. 